Pacific

Journal of

Mathematics

NONRATIONAL NODAL QUARTIC THREEFOLDS

IVAN Cheltsov 


\title{
NONRATIONAL NODAL QUARTIC THREEFOLDS
}

\author{
IVAN CHELTSOV
}

\begin{abstract}
It is known that the $\mathbb{Q}$-factoriality of a nodal quartic 3-fold in $\mathbb{P}^{4}$ implies its nonrationality. We prove that a nodal quartic 3-fold with at most 8 nodes is $\mathbb{Q}$-factorial, while one with 9 nodes is not $\mathbb{Q}$-factorial if and only if it contains a plane. There are nonrational non- $\mathbb{Q}$-factorial nodal quartic 3-folds. In particular, we prove the nonrationality of a general non- $\mathbb{Q}$-factorial nodal quartic 3-fold that contains either a plane or a smooth del Pezzo surface of degree 4.
\end{abstract}

\section{Introduction}

All varieties are assumed to be projective, normal and defined over $\mathbb{C}$.

Let $X \subset \mathbb{P}^{4}$ be a nodal quartic 3-fold, that is, a hypersurface of degree 4 whose singular points are ordinary double points. Then $X$ is a Fano 3-fold with terminal singularities and satisfies $-\left.K_{X} \sim \mathcal{O}_{\mathbb{P}^{4}}(1)\right|_{X}$. The following result is Theorem 2 in [Mella 2004] (see also [Iskovskih and Manin 1971; Pukhlikov 1988]):

Theorem 1. Suppose $X$ is $\mathbb{Q}$-factorial. Then $X$ is not birational to either a conic bundle, a fibration in rational surfaces, or a Fano 3-fold of Picard rank 1 with terminal $\mathbb{Q}$-factorial singularities that is not biregular to $X$.

In this paper we prove:

Theorem 2. If $|\operatorname{Sing} X| \leq 8$, then $X$ is $\mathbb{Q}$-factorial.

Corollary 3. Nodal quartic 3-folds with at most 8 nodes are nonrational.

The conditions of Theorem 2 cannot be weakened:

Example 4. If $X$ is a sufficiently general quartic 3-fold containing a two-dimensional linear subspace $\Pi \subset \mathbb{P}^{4}$, then $X$ is nodal, non- $\mathbb{Q}$-factorial, and has 9 nodes, which are the intersection of two cubic curves in the plane $\Pi$.

However, we prove:

Theorem 5. Suppose that $|\operatorname{Sing} X|=9$. The quartic $X$ is $\mathbb{Q}$-factorial if and only if it does not contain any two-dimensional linear subspace of $\mathbb{P}^{4}$.

MSC2000: 14E08, 14J30, 14J45, 14J70, 14M20.

Keywords: quartic threefold, nodal variety, Fano variety, del Pezzo surface, $\mathbb{Q}$-factorial.

This work is partially supported by CRDF grant RUM1-2692MO-05. 
A general nodal quartic 3-fold with 9 nodes is $\mathbb{Q}$-factorial. A posteriori, the non$\mathbb{Q}$-factoriality of the quartic $X$ does not necessarily imply its rationality. Indeed, we prove the following result (compare with [Mella 2004, Remark 3]):

Theorem 6. A very general quartic 3-fold X containing a two-dimensional linear subspace of $\mathbb{P}^{4}$ is nonrational.

Nonetheless, rational nodal quartic 3-folds do exist:

Example 7 [Petterson 1998]. Any general determinantal quartic 3-fold $X$ is nodal, rational, non- $\mathbb{Q}$-factorial, and satisfies $|\operatorname{Sing} X|=20$.

Remark 8. The quartic $X$ cannot have more than 45 nodes [Varchenko 1983; Friedman 1986]. It is shown in [de Jong et al. 1990] that there is a unique nodal quartic 3 -fold $\mathscr{B}_{4}$ with 45 nodes, which can be given by the equation

$$
w^{4}-w\left(x^{3}+y^{3}+z^{3}+t^{3}\right)+3 x y z t=0
$$

in $\mathbb{P}^{4} \cong \operatorname{Proj}(\mathbb{C}[x, y, z, t, w])$. It is known as the Burkhardt quartic [Burkhardt 1891; 1892; Todd 1936; Baker 1946; Finkelberg 1989; Petterson 1998]. This quartic is determinantal and, moreover, is the unique invariant of degree 4 of the simple group PSp $\left(4, \mathbb{Z}_{3}\right)$ of order 25920 [van der Geer 1987; Hunt 1996; Hoffman and Weintraub 2001; Hulek and Sankaran 2002]. The nodes of $\mathscr{B}_{4}$ correspond to the 45 tritangents of a smooth cubic surface, and the Weyl group of $E_{6}$ is a nontrivial extension of $\operatorname{PSp}\left(4, \mathbb{Z}_{3}\right)$ by $\mathbb{Z}_{2}$.

For a given variety, one of the most substantial questions is to decide whether it is rational. This question has been considered in depth for smooth 3-folds [Iskovskih and Manin 1971; Clemens and Griffiths 1972; Beauville 1977; Tyurin 1980; Sarkisov 1980; Shokurov 1983; Alekseev 1987; Corti 1995; Pukhlikov 1998; Iskovskikh and Prokhorov 1999; Corti 2000]. However, relatively mild singularities can force a 3-fold to be rational. For example, with a few exceptions, all canonical Gorenstein Fano 3-folds having a non-cDV point are rational [Prokhorov 2004]. In the non-Gorenstein case the situation is different [Corti et al. 2000; Cheltsov 1997, 2004]. Hence, the rationality of nodal 3-folds is a natural topic [Pukhlikov 1988; Grinenko 1998a, 1998b; Mella 2004; Cheltsov and Park 2004].

Remark 9. Every nodal hypersurface in $\mathbb{P}^{4}$ of degree at least 5 is nonrational. Every quadric 3-fold in $\mathbb{P}^{4}$ is rational. A nodal cubic 3-fold in $\mathbb{P}^{4}$ is nonrational if and only if it is smooth. See [Clemens and Griffiths 1972, Theorem 13.12].

There are non- $\mathbb{Q}$-factorial nodal quartic 3-folds that do not contain any twodimensional linear subspaces of $\mathbb{P}^{4}$; see [Mella 2004; Ellia and Franco 2000]. 
Example 10. Consider a sufficiently general quartic 3-fold $X \subset \mathbb{P}^{4}$, passing through a smooth quadric surface $Q \subset \mathbb{P}^{4}$. The quartic $X$ can be given by the equation

$$
a_{2}(x, y, z, t, w) h_{2}(x, y, z, t, w)=b_{3}(x, y, z, t, w) g_{1}(x, y, z, t, w)
$$

in $\operatorname{Proj}(\mathbb{C}[x, y, z, t, w])$. Here, $a_{2}, h_{2}, b_{3}$ and $g_{1}$ are homogeneous polynomials of degrees 2, 2, 3 and 1 respectively; the quadric $Q \subset \mathbb{P}^{4}$ is given by the equations $h_{2}=g_{1}=0$. The 3 -fold $X$ is nodal and non- $\mathbb{Q}$-factorial, and it has 12 nodes given by $h_{2}=g_{1}=a_{2}=b_{3}=0$. Introducing a new variable $\alpha=a_{2} / g_{1}$, we obtain a commutative diagram

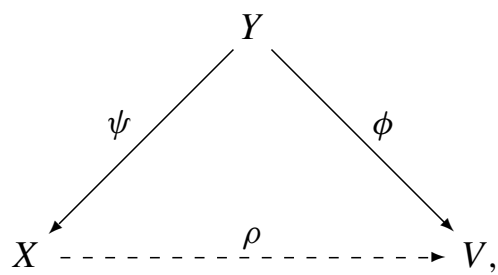

where $\rho$ is a birational map, $\phi$ is an extremal divisorial contraction [Corti 1995, §3.3.1], the morphism $\psi: Y \rightarrow X$ is a flopping contraction [Kollár 1989], and $V$ is a complete intersection

$$
\alpha g_{1}(x, y, z, t, w)-a_{2}(x, y, z, t, w)=\alpha h_{2}(x, y, z, t, w)-b_{3}(x, y, z, t, w)=0
$$

in $\mathbb{P}^{5}$. Often $\rho$ is called an unprojection of $X$ [Reid 2001]. The variety $V$ is smooth outside a point $P=(0: 0: 0: 0: 0: 1)$, which is a node. The morphism $\phi$ contracts the surface $\mathbb{P}^{1} \times \mathbb{P}^{1}$ to $P$, while $\psi$ contracts the images of 12 lines on $V$ passing through $P$ to the nodes of $X$. It is unknown whether $X$ is rational [Iskovskikh and Pukhlikov 1996; Corti 2000].

There exist nonrational non- $\mathbb{Q}$-factorial nodal quartic 3-folds in $\mathbb{P}^{4}$ that contain neither planes nor quadric surfaces. In particular, we will prove:

Theorem 11. If $X \subset \mathbb{P}^{4}$ is a sufficiently general quartic 3-fold that contains a smooth del Pezzo surface $S \subset \mathbb{P}^{4}$ of degree 4, then $X$ is nodal, non- $\mathbb{Q}$-factorial and nonrational, and has $|\operatorname{Sing} X|=16$.

The varieties in Theorems 6 and 11 are the only known examples of nodal, nonrational and non- $\mathbb{Q}$-factorial quartic 3-folds. We note that the degeneration technique [Clemens 1975; Beauville 1977; Tyurin 1980; Kollár 1996], together with either Theorem 6 or Theorem 11, gives another proof that a very general smooth quartic 3-fold is nonrational.

Remark 12. There are only a few examples of unirational smooth quartic 3-folds [Iskovskih and Manin 1971; Marchisio 2000], and it is unknown whether a generic 
quartic 3-fold is unirational. However, the quartics in Theorems 6 and 11 are birational to del Pezzo fibrations of degree 3 or 4, which implies that the quartics in Theorems 6 and 11 are unirational [Manin 1967].

Theorems 2 and 5 can be considered part of the following conjecture:

Conjecture 13. Let $V \subset \mathbb{P}^{4}$ be a nodal hypersurface. If either

- $|\operatorname{Sing} V|<(\operatorname{deg} V-1)^{2}$,

- $|\operatorname{Sing} V|<2(\operatorname{deg} V-1)(\operatorname{deg} V-2)$ and $V$ does not contain planes, or

- $|\operatorname{Sing} V| \leq 2(\operatorname{deg} V-1)(\operatorname{deg} V-2)$ and $V$ does not contain planes or quadrics, then $V$ is $\mathbb{Q}$-factorial.

We note that an analogue to Conjecture 13 for smooth surfaces on a nodal hypersurface in $\mathbb{P}^{4}$ is proved in [Ciliberto and Di Gennaro 2004]. It is easy to see that Conjecture 13 holds for quadrics and cubics [Finkelnberg and Werner 1989].

\section{The proof of Theorems 2 and 5}

The $\mathbb{Q}$-factoriality of nodal 3-folds is studied in [Clemens 1983; Schoen 1985; Werner 1987; Dimca 1990; Borcea 1990; Endraß 1999; Cynk 2001].

Let $X \subset \mathbb{P}^{4}$ be a nodal quartic 3-fold. It is well known [Werner 1987; Dimca 1990; Cynk 2001] that the following conditions are equivalent:

- The quartic $X$ is $\mathbb{Q}$-factorial.

- Every Weil divisor on $X$ is a Cartier divisor.

- Every Zariski local ring of the quartic $X$ is UFD, that is, $X$ is factorial.

- The group $H_{4}(X, \mathbb{Z})$ is generated by the class of a hyperplane section.

- $\operatorname{dim} H_{4}(X, \mathbb{Z})=\operatorname{dim} H^{2}(X, \mathbb{Z})=1$.

- The nodes of $X$ impose independent linear conditions on cubic hypersurfaces in $\mathbb{P}^{4}$.

Suppose $X$ does not contain planes and $|\operatorname{Sing} X| \leq 9$. We show that the nodes of the quartic $X$ impose independent linear conditions on cubic hypersurfaces in $\mathbb{P}^{4}$.

Definition 14. The points of a set $\Gamma \subset \mathbb{P}^{4}$ are in general position if

- at most 3 points of $\Gamma$ can lie on a line,

- at most 6 points of $\Gamma$ can lie on a conic, and

- at most 8 points of $\Gamma$ can lie on a plane.

Proposition 15. The nodes of the quartic $X$ are in general position. 
Proof. Let $L \subset \mathbb{P}^{4}$ be a line and $\Pi \subset \mathbb{P}^{4}$ a sufficiently general two-dimensional linear subspace passing through $L$. We have $\Pi \not \subset X$, and $\Pi \cap X=L \cup S$ for some plane cubic curve $S$. Moreover,

\section{Sing $X \cap L \subset L \cap S$,}

but $|L \cap S| \leq 3$. Thus, at most 3 nodes of the quartic $X$ can lie on a line in $\mathbb{P}^{4}$.

Let $C \subset \mathbb{P}^{4}$ be a smooth conic and $Y \subset \mathbb{P}^{4}$ a sufficiently general two-dimensional quadric cone over the conic $C$. We have $Y \not \subset X$, and $Y \cap X=C \cup R$ for some curve $R$ of degree 6 . As above, we have the inclusion

$$
\text { Sing } X \cap C \subset C \cap R \text {. }
$$

However, the curves $C$ and $R$ lie in the smooth locus of $Y$ and the intersection $C \cdot R$ in $Y$ equals 6 . Thus, the inequality $|C \cap R| \leq 6$ holds, and at most 6 nodes of the 3 -fold $X$ can lie on a smooth conic in $\mathbb{P}^{4}$.

Let $\Sigma \subset \mathbb{P}^{4}$ be a plane. The intersection $T=\Sigma \cap X$ is a possibly reducible and nonreduced plane quartic, and $\operatorname{Sing} X \cap \Sigma \subset \operatorname{Sing} T$. In case $T$ is nonreduced, we have $|\operatorname{Sing} X \cap \Sigma| \leq 6$, as we already proved that at most 3 nodes of $X$ can lie on a line and at most 6 nodes can lie on a conic. Moreover, $|\operatorname{Sing} T| \leq 6$ whenever $T$ is reduced. Therefore, at most 6 nodes of $X$ can lie on a plane in $\mathbb{P}^{4}$.

Proposition 16. Let $\Pi \subset \mathbb{P}^{4}$ be a two-dimensional linear subspace such that Sing $X$ is contained in $\Pi$. The nodes of $X$ impose independent linear conditions both on cubic curves in $\Pi \cong \mathbb{P}^{2}$ and on cubic hypersurfaces in $\mathbb{P}^{4}$.

Proof. We must show that, for any subset $\Sigma \subsetneq \operatorname{Sing} X$ and any point $p \in \operatorname{Sing} X \backslash \Sigma$, there exist a cubic curve in $\Pi$ and a cubic hypersurface in $\mathbb{P}^{4}$ passing through the points of $\Sigma$ but not through $p$. Let $\pi: V \rightarrow \Pi$ be a blow-up of points in $\Sigma$. Then, owing to Proposition 15, $V$ is a weak del Pezzo surface of degree $9-|\Sigma| \geq 2$.

The linear system $\left|-K_{V}\right|$ does not have base points [Demazure 1980, §IV, Theorem 1; Bese 1983, Theorem 2]. Thus, there exists a curve $C$ in $\left|-K_{V}\right|$ that does not pass through the point $\pi^{-1}(p)$. The cubic curve $\pi(C)$ passes through all the points of $\Sigma$ but not through $p$. Let $Y$ be a cone in $\mathbb{P}^{4}$ over $\pi(C)$, with vertex on a sufficiently general line of $\mathbb{P}^{4}$. The cubic hypersurface $Y$ passes through all the points of $\Sigma$ but not through $p$.

Lemma 17 [Cheltsov and Park 2004]. Let $\Delta \subset \mathbb{P}^{n}$ be a subset and $p \in \mathbb{P}^{n} \backslash \Delta a$ point such that $\{p\} \cup \Delta \subset \mathbb{P}^{n}$ is not contained in any linear subspace of dimension $r$. There exists a linear subspace $H \subset \mathbb{P}^{n}$ of dimension $r$ that contains at least $r+1$ points of $\Delta$ but not $p$.

Proof. We prove the claim by induction on $n$. For $n=2$ the claim is trivial. Suppose that $n>2$ and $r<n$. By assumption, there are $r+1$ points $\left\{q_{1}, \ldots, q_{r+1}\right\} \subset \Delta$ such that their linear span $T$ has dimension $r$. 
We assume $p \in T$, since otherwise we are done. There is a point $q \in \Delta \backslash T$, because $\{p\} \cup \Delta \subset \mathbb{P}^{n}$ is not contained in any linear subspace of dimension $r$. By induction, there exists a linear subspace $S \subset T$ of dimension $r-1$ that contains $r$ points from among $\left\{q_{1}, \ldots, q_{r+1}\right\}$ but not $p$.

Consider a cone $H \subset \mathbb{P}^{n}$ over $T$ with vertex $q$. The cone $H$ is a linear subspace of dimension $r$ that contains at least $r+1$ points of $\Delta$ but not $p$.

Proposition 18. Let $\Gamma \subset \mathbb{P}^{4}$ be a hyperplane such that $\operatorname{Sing} X$ is contained in $\Gamma$. The nodes of $X$ impose independent linear conditions both on cubic surfaces in $\Gamma \cong \mathbb{P}^{3}$ and on cubic hypersurfaces in $\mathbb{P}^{4}$.

Proof. Let $\Sigma \subsetneq \operatorname{Sing} X$ be any subset and let $p \in \operatorname{Sing} X \backslash \Sigma$ be a point. We must show that there is a cubic surface in $\Gamma$ and a cubic hypersurface in $\mathbb{P}^{4}$ passing through $\Sigma$ and not passing through $p$. As in the proof of Proposition 16, it is enough to find a cubic surface in $\Gamma$ that passes through all the points of $\Sigma$ but not through $p$. A general cone over such a cubic surface will then give a cubic hypersurface in $\mathbb{P}^{4}$ passing through all the points of $\Sigma$ and not passing through $p$.

Without loss of generality, we can assume that $\mid$ Sing $X|=| \Sigma \mid+1=9$.

Let $r \geq 2$ be the maximal number of points of $\Sigma$ that, together with $p$, belong to a two-dimensional linear subspace $\Pi$ in $\Gamma$. Then, by Proposition $15, r \leq 7$. Write

$$
\Sigma=\left\{p_{1}, \ldots, p_{8}\right\}
$$

so that the points $p_{1}, \ldots, p_{r}$, together with $p$, are contained in the plane $\Pi$. The points $p$ and $p_{1}, \ldots, p_{r}$ do not lie on any one line, since otherwise we could find a hyperplane in $\Gamma$ containing more than $r$ points of $\Sigma$. We prove the statement case by case.

Suppose $r=2$. Divide the set $\Sigma$ into three, possibly overlapping, subsets such that each subset contains three points of $\Sigma$ and that their union is the whole $\Sigma$. The hyperplane in $\Gamma$ generated by each subset does not contain $p$, because $r=2$. Hence the union of these three hyperplanes is the required cubic surface.

Suppose $r=3$. By Lemma 17, we can find three points of $\Sigma$ outside $\Pi$, say $p_{4}, p_{5}, p_{6}$, such that they generate a hyperplane in $\Gamma$ not passing though $p$. By Proposition 15, the four points $\left\{p, p_{1}, p_{2}, p_{3}\right\}$ do not lie on any one line. Therefore there is a line passing through two points of the set $\left\{p_{1}, p_{2}, p_{3}\right\}$, say through $p_{1}$ and $p_{2}$, and not passing through $p$. The union of a hyperplane passing through $p_{4}, p_{5}, p_{6}$, a hyperplane passing through $p_{7}, p_{1}, p_{2}$, and a sufficiently general hyperplane passing through $p_{3}$ and $p_{8}$ gives a cubic surface in $\Gamma \cong \mathbb{P}^{3}$ that passes through all the points of $\Sigma$ but not through $p$.

Suppose $r=4$. There are two lines in $\Pi$, say $L_{1}$ and $L_{2}$, such that the line $L_{1}$ contains $p_{1}$ and $p_{2}$, the line $L_{2}$ contains $p_{3}$ and $p_{4}$, and neither line passes through $p$. At most two points among $\left\{p_{5}, p_{6}, p_{7}, p_{8}\right\}$ lie on a line passing through $p$. 
Therefore, there are two points, say $p_{5}$ and $p_{6}$, such that the line passing through $p_{5}$ and $p_{6}$ does not pass through $p$. The union of a hyperplane passing through $L_{1}$ and $p_{7}$, a hyperplane passing through $L_{2}$ and $p_{8}$, and a sufficiently general hyperplane passing through $p_{5}$ and $p_{6}$ gives the required cubic surface.

Suppose $r=5$. There are two lines in $\Pi$, say $L_{1}$ and $L_{2}$, such that $p \notin L_{1} \cup L_{2}$ and $L_{1} \cup L_{2}$ contains four points of $\Sigma \cap \Pi$, say $p_{1}, p_{2}, p_{3}$ and $p_{4}$. The union of a hyperplane passing through $L_{1}$ and $p_{7}$, a hyperplane passing through $L_{2}$ and $p_{8}$, and a sufficiently general hyperplane passing through $p_{5}$ and $p_{6}$ gives a cubic surface in $\Gamma$ passing through all the points of $\Sigma$ but not through $p$.

Suppose $r=6$. Now we have six points in $\Sigma \cap \Pi$ and two points, say $p_{7}$ and $p_{8}$, of $\Sigma$ outside $\Pi$. By Proposition 16, we can find a cubic curve $C$ on $\Pi$ that passes through the points of $\Sigma \cap \Pi$ but not through $p$. A sufficiently general hyperplane in $\Gamma$ passing through the points $p_{7}$ and $p_{8}$ meets the curve $C$ in three points. Let $q$ and $q^{\prime}$ be two of those points, and let $O$ be an intersection point of the lines $\left\langle p_{7}, q\right\rangle$ and $\left\langle p_{8}, q^{\prime}\right\rangle$. Then the cone in $\Gamma$ over the cubic curve $C$ with vertex $O$ is a cubic surface that passes through all the points of $\Sigma$ but not through $p$.

Suppose $r=7$. By Proposition 16, we can find a cubic curve $C$ on $\Pi$ that passes through the seven points of $\Sigma \cap \Pi$ and does not pass through the point $p$. The cone in $\Gamma \cong \mathbb{P}^{3}$ over the cubic curve $C$ with vertex $p_{8}$ is a cubic surface that passes through $\Sigma$ but not through $p$.

Proposition 19. The nodes of $X$ impose independent linear conditions on cubic curves in $\mathbb{P}^{4}$.

Proof. We must show that, for any subset $\Sigma \subsetneq \operatorname{Sing} X$ and any point $p \in \operatorname{Sing} X \backslash \Sigma$, there exists a cubic hypersurface in $\mathbb{P}^{4}$ passing through $\Sigma$ but not through $p$.

Without loss of generality, we may assume that $\mid$ Sing $X|=| \Sigma \mid+1=9$.

Let $r \geq 3$ be the maximal number of points in $\Sigma$ that, together with $p$, belong to a hyperplane $\Xi \subset \mathbb{P}^{4}$. By Proposition 18, we may assume $r \leq 7$. Write

$$
\Sigma=\left\{p_{1}, \ldots, p_{8}\right\}
$$

so that the points $p_{1}, \ldots, p_{r}$, together with the point $p$, are contained in $\Xi$. We prove the claim case by case.

The points $p$ and $p_{1}, \ldots, p_{r}$ do not belong to a two-dimensional linear subspace in $\mathbb{P}^{4}$, since otherwise we could find a hyperplane passing through $r+1$ points of the set $\Sigma$.

Suppose $r=3$. Divide the set $\Sigma$ into three, possibly overlapping, subsets such that each subset contains exactly four points of $\Sigma$. The hyperplane generated by each subset does not contain the point $p$, because $r=3$. The union of these three hyperplanes is the required cubic hypersurface. 
Suppose $r=4$. There are two lines $L_{1}$ and $L_{2}$ in $\Xi$ such that the line $L_{1}$ passes through $p_{1}$ and $p_{2}$, the line $L_{2}$ passes through $p_{3}$ and $p_{4}$, and neither line passes through $p$. There are at most two points of $\left\{p_{5}, p_{6}, p_{7}, p_{8}\right\}$ that lie on a line containing $p$. Hence, there are two points, say $p_{5}$ and $p_{6}$, such that the line passing through $p_{5}$ and $p_{6}$ does not pass through $p$. The union of two sufficiently general hyperplanes, one passing through $L_{1}$ and $p_{7}$, the other through $L_{2}$ and $p_{8}$, and a sufficiently general hyperplane passing through the points $p_{5}$ and $p_{6}$ gives the required cubic hypersurface in $\mathbb{P}^{4}$.

Suppose $r=5$. As in the previous case, there are two lines $L_{1}$ and $L_{2}$ in $\Xi$ such that the line $L_{1}$ passes through $p_{1}$ and $p_{2}$, the line $L_{2}$ passes through $p_{3}$ and $p_{4}$, and neither line passes through $p$. The union of two general hyperplanes, one passing through the $L_{1}$ and $p_{7}$, one through $L_{2}$ and $p_{8}$, and a sufficiently general hyperplane passing through the points $p_{5}$ and $p_{6}$ gives a cubic hypersurface in $\mathbb{P}^{4}$ that passes through all the points of $\Sigma$ but not through $p$.

Suppose $r=6$. There are six points in $\Sigma \cap \Xi$ and two points, say $p_{7}$ and $p_{8}$, of $\Sigma$ outside $\Xi$. By Proposition 18, there is a cubic surface $S \subset \Xi$ that passes through the six points of $\Sigma \cap \Xi$ and does not pass through $p$. A general two-dimensional linear subspace passing through the points $p_{7}$ and $p_{8}$ meets $S$ in three different points. From these, choose two points $q$ and $q^{\prime}$. Let $O$ be an intersection of the lines $\left\langle p_{7}, q\right\rangle$ and $\left\langle p_{8}, q^{\prime}\right\rangle$. The required cubic hypersurface is the cone in $\mathbb{P}^{4}$ over the cubic surface $S$ with vertex $O$.

Suppose $r=7$. By Proposition 18, we can find a cubic surface $S \subset \Xi$ that passes through the seven points of $\Sigma \cap \Pi$ and does not pass through $p$. The cone in $\mathbb{P}^{4}$ over $S$ with vertex $p_{8}$ passes through all the points of $\Sigma$ but not through $p$.

This concludes the proof of Theorems 2 and 5. The same method can be applied to any nodal hypersurface in $\mathbb{P}^{4}$. The following result is implied by Theorem 24 (see [Werner 1987; Dimca 1990; Ciliberto and Di Gennaro 2004]).

Theorem 20. A nodal hypersurface $V \subset \mathbb{P}^{4}$ is $\mathbb{Q}$-factorial whenever

$$
|\operatorname{Sing} V| \leq 2 \operatorname{deg} V-4 \text {. }
$$

This bound on nodes is not sharp, except for hyperquadrics.

\section{The proof of Theorem 11}

Let $X \subset \mathbb{P}^{4}$ be a sufficiently general (that is, from the complement of a Zariski closed subset in moduli) quartic 3-fold containing a smooth del Pezzo surface $S \subset$ $\mathbb{P}^{4}$ of degree 4 . The quartic $X$ can be given by the equation

$$
a_{2}(x, y, z, t, w) h_{2}(x, y, z, t, w)+b_{2}(x, y, z, t, w) g_{2}(x, y, z, t, w)=0
$$


in $\operatorname{Proj}(\mathbb{C}[x, y, z, t, w])$. Here, $a_{2}, b_{2}, h_{2}$ and $g_{2}$ are homogeneous polynomials of degree 2 such that $S$ is defined by the equations $h_{2}=g_{2}=0$. The quartic $X$ is nodal and non- $\mathbb{Q}$-factorial; it has 16 nodes given by the equations $h_{2}=g_{2}=a_{2}=b_{2}=0$.

Lemma 21. The divisor class group $\mathrm{Cl}(X)$ is $\mathbb{Z} \oplus \mathbb{Z}$.

Proof. Let $f: U \rightarrow \mathbb{P}^{4}$ be a blow-up of the surface $S$, let $E$ be an exceptional divisor of the birational map $f$, and let $H=f^{*}\left(\mathcal{O}_{\mathbb{P}^{4}}(1)\right)$. The linear system $|2 H-E|$ does not have base points, because the del Pezzo surface $S \subset \mathbb{P}^{4}$ is a complete intersection of two quadrics. In particular, the divisor $2 H-E$ is nef and the divisor $4 H-E$ is ample.

Let $\tilde{X} \subset U$ be a proper transform of the quartic $X$. Then $\tilde{X}$ is rationally equivalent to the divisor $4 H-E$ on the 4-fold $U$. The restriction $\left.f\right|_{\tilde{X}}: \tilde{X} \rightarrow X$ is a small resolution and $\tilde{X}$ is smooth. Therefore, by the Lefschetz theorem [Andreotti and Frankel 1959; Bott 1959],

$$
H^{2}(\tilde{X}, \mathbb{Z}) \cong H^{2}(U, \mathbb{Z}) \cong \mathbb{Z} \oplus \mathbb{Z},
$$

which implies the claim of the lemma.

The pencil generated by the quadrics $a_{2}=0$ and $b_{2}=0$ cuts on $X$ the del Pezzo surface $S$ together with a pencil $M$ whose general element is a smooth del Pezzo surface of degree 4. Let $\tau: V \rightarrow X$ be a small resolution such that the pencil $\mathscr{H}=\tau^{-1}(\mathcal{M})$ does not have base points. We have

$$
V=\operatorname{Proj}\left(\bigoplus_{i \geq 0} O_{X}(-S)^{\otimes i}\right),
$$

and $\tau$ is a natural projection to $X$ [Kawamata 1988]. The 3-fold $V$ is smooth and projective, we have $\operatorname{Pic}(V)=\mathbb{Z} \oplus \mathbb{Z}$, and the pencil $\mathscr{H}$ gives a morphism

$$
\xi: V \rightarrow \mathbb{P}^{1}
$$

whose general fiber is a del Pezzo surface of degree 4 .

Corollary 22 [Alekseev 1987; Iskovskikh 1996a]. The 3-fold $V$ is birational to a conic bundle.

The generality of the choice of $X$ implies that $\xi$ is standard in the sense of [Alekseev 1987]; that is, every fiber of $\xi$ is normal and $\operatorname{Pic}(V)=\mathbb{Z} \oplus \mathbb{Z}$.

Theorem 23 [Alekseev 1987, Theorem 2]. Let $\gamma: Y \rightarrow \mathbb{P}^{1}$ be a standard del Pezzo fibration of degree 4. If the topological Euler characteristic of $Y$ is not $0,-4$ or -8 , then $Y$ is nonrational.

Therefore, in order to prove Theorem 11, we must calculate the topological Euler characteristic of the 3-fold $V$. 
Theorem 24 [Cynk 2001, Theorem 2]. Let $W$ be a projective smooth 4-fold, and $Y$ an ample reduced and irreducible divisor on $W$ such that the only singularities of $Y$ are nodes and such that

$$
h^{2}\left(\Omega_{W}^{1}\right)=h^{3}\left(\Omega_{W}^{1} \otimes \mathscr{O}_{W}(-Y)\right)=h^{1}\left(\mathbb{O}_{W}\right)=h^{2}\left(\mathscr{O}_{W}\right)=0 .
$$

If $\tilde{Y}$ is a small resolution of $Y$, then

$$
\begin{aligned}
h^{1}\left(\mathcal{O}_{\tilde{Y}}\right) & =h^{2}\left(\mathcal{O}_{\tilde{Y}}\right)=0, \\
h^{1}\left(\Omega_{\tilde{Y}}^{1}\right) & =h^{1}\left(\Omega_{W}^{1}\right)+\delta, \\
h^{2}\left(\Omega_{\tilde{Y}}^{1}\right) & =h^{0}\left(K_{W} \otimes \mathcal{O}_{W}(2 Y)\right)+h^{3}\left(\mathcal{O}_{W}\right)-h^{0}\left(K_{W} \otimes \mathcal{O}_{W}(Y)\right) \\
& \quad-h^{3}\left(\Omega_{W}^{1}\right)-h^{4}\left(\Omega_{W}^{1} \otimes \mathcal{O}_{W}(-Y)\right)-|\operatorname{Sing} Y|+\delta,
\end{aligned}
$$

where $\delta$ is the number of dependent equations that are imposed on the global sections of the line bundle $K_{W} \otimes \mathrm{O}_{W}(2 Y)$ by the vanishing at the nodes of $Y$; that is, $\delta$ is the defect of the 3-fold $Y$.

The topological Euler characteristic $\chi(V)$ of the 3 -fold $V$ is $6-2 h^{2}\left(\Omega_{V}^{1}\right)$. The twisted Euler exact sequence and Serre duality imply that $h^{3}\left(\Omega_{\mathbb{p}^{4}}^{1} \otimes \mathcal{O}_{\mathbb{P}^{4}}(-4)\right)=0$ and $h^{4}\left(\Omega_{\mathbb{P}^{4}}^{1} \otimes \mathcal{O}_{\mathbb{P}^{4}}(-4)\right)=5$. Thus, by Theorem 24 ,

$$
h^{2}\left(\Omega_{V}^{1}\right)=h^{0}\left(\mathbb{O}_{\mathbb{P}^{4}}(3)\right)-h^{3}\left(\Omega_{\mathbb{P}^{4}}^{1}\right)-h^{4}\left(\Omega_{\mathbb{P}^{4}}^{1} \otimes \mathbb{O}_{\mathbb{P}^{4}}(-4)\right)-|\operatorname{Sing} X|+1=15 .
$$

It follows that $\chi(V)=-24$. By Theorem 23, the quartic 3-fold $X$ is nonrational, which proves Theorem 11.

\section{The proof of Theorem 6}

Let $X \subset \mathbb{P}^{4}$ be a very general (that is, from the complement of a countable union of Zariski closed subsets in moduli) quartic 3-fold containing a plane $\Pi \subset \mathbb{P}^{4}$. The quartic $X$ can be given by the equation

$$
x h_{3}(x, y, z, t, w)+y g_{3}(x, y, z, t, w)=0
$$

in $\operatorname{Proj}(\mathbb{C}[x, y, z, t, w])$. Here, $h_{3}$ and $g_{3}$ are homogeneous polynomials of degree 3 , while the plane $\Pi$ is defined by the equations $x=y=0$. The quartic $X$ is nodal and has 9 nodes given by

$$
x=y=h_{3}=g_{3}=0 .
$$

Lemma 25. The divisor class group $\mathrm{Cl}(X)$ is $\mathbb{Z} \oplus \mathbb{Z}$.

Proof. By Theorem 24, the lemma's assertion is equivalent to the statement nodes of the quartic $X$ imposes 8 independent linear conditions on cubic hypersurfaces in $\mathbb{P}^{4}$. That is, we must show that the defect of $X$ is 1 . 
During the proof of Theorem 2, we showed that any 8 nodes of $X$ impose 8 independent linear conditions on cubic hypersurfaces in $\mathbb{P}^{4}$. The nodes of $X$ cannot impose 9 independent linear conditions on cubic hypersurfaces in $\mathbb{P}^{4}$, because in this case, by Theorem 24 , the 3 -fold $X$ must be $\mathbb{Q}$-factorial, which is absurd.

Theorem 26 [Kollár 1996, §IV, Theorem 1.8.3]. Let $\xi: Y \rightarrow Z$ be a flat proper morphism with irreducible and reduced geometric fibers. There are countably many closed subvarieties $Z_{i} \subset Z$ such that, for an arbitrary closed point $s \in Z$, the fiber $\xi^{-1}(s)$ is ruled if and only if $s \in \bigcup Z_{i}$.

Consider a sufficiently general quartic 3 -fold $V \subset \mathbb{P}^{4}$, given by the equation

$$
x \bar{h}_{3}(x, y, z, t, w)+y \bar{g}_{3}(x, y, z, t, w)=0,
$$

such that

$$
\bar{h}_{3}(x, y, z, t, w)=x a_{2}(x, y, z, t, w)+y b_{2}(x, y, z, t, w)+f_{1}(z, t, w) h_{2}(z, t, w)
$$

and

$\bar{g}_{3}(x, y, z, t, w)=x c_{2}(x, y, z, t, w)+y d_{2}(x, y, z, t, w)+f_{1}(z, t, w) g_{2}(z, t, w)$,

where $a_{2}, b_{2}, c_{2}, d_{2}, h_{2}$ and $g_{2}$ are homogeneous polynomials of degree 2 , and $f_{1}$ is a homogeneous polynomial of degree 1 . The quartic $V$ contains the plane $\Pi$. The singularities of the 3 -fold $V$ consist of 4 nodes given by the equations

$$
x=y=h_{2}=g_{2}=0
$$

and a single double line $L \subset \Pi$ given by the equations $x=y=f_{1}=0$.

Remark 27. The resolution of singularities of $V$ is rationally connected by [Kollár 1996, §IV, Corollary 5.7.1]. Hence, the 3-fold $V$ is rational if and only if it is ruled. However, the 3-fold $V$ is a flat degeneration of the 3-fold $X$. Thus, by Theorem 26, the nonrationality of $V$ implies the nonrationality of $X$.

To prove Theorem 6 it is enough to prove the nonrationality of $V$.

Remark 28. The nonrationality of a sufficiently general quartic 3-fold with a double line is proved in [Conte and Murre 1977] by using the method of the intermediate Jacobian [Clemens and Griffiths 1972; Beauville 1977; Tyurin 1980].

Let $\pi: U \rightarrow \mathbb{P}^{4}$ be a blow-up of the line $L \subset \mathbb{P}^{4}$, let $E$ be an exceptional divisor of the birational morphism $\pi$, and let $\bar{V} \subset U$ be a proper transform of the 3-fold $V$. The linear system

$$
\left|\pi^{*}\left(\mathbb{O}_{\mathbb{P}^{4}}(1)\right)-E\right|
$$

does not have base points and gives a $\mathbb{P}^{2}$-bundle $\psi: U \rightarrow \mathbb{P}^{2}$. 
We have the commutative diagram

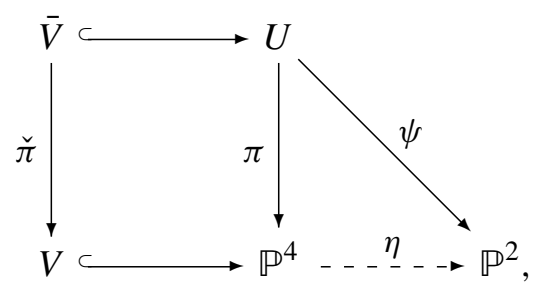

where $\eta$ is the projection from the line $L$. The 3 -fold $\bar{V}$ is smooth in the neighborhood of the exceptional divisor $E$, while the singularities of $\bar{V}$ consist of 4 nodes that are the images of the nodes of $V$.

For a point $x \in L$, the intersection

$$
\pi^{-1}(x) \cap \bar{V} \subset \pi^{-1}(x) \cong \mathbb{P}^{2}
$$

is a smooth conic if $x$ is not a zero of $h_{2}$ or $g_{2}$, and is a union of two different lines otherwise; that is, there are 4 reducible fibers of the morphism $\left.\pi\right|_{E \cap \bar{V}}$.

Let $\bar{\Pi} \subset U$ be a proper transform of $\Pi$. Then $\psi(\bar{\Pi})=O$ is a point. The restriction

$$
\left.\psi\right|_{\bar{V}}: \bar{V} \rightarrow \mathbb{P}^{2}
$$

is a morphism whose fibers over the points in $\mathbb{P}^{2} \backslash O$ are conics, while over $O$ is the surface $\bar{\Pi} \subset \bar{V}$.

Let $\gamma: W \rightarrow U$ be a blow-up of $\bar{\Pi}$, let $G$ be a $\gamma$-exceptional divisor, and let $\tilde{V} \subset W$ be a proper transform of $\bar{V}$. The linear system $\left|\gamma^{*}\left(\pi^{*}\left(\mathscr{O}_{\mathbb{P}^{4}}(1)\right)-E\right)-G\right|$ has no base points, while the linear system $\left|\gamma^{*}\left(2\left(\pi^{*}\left(\mathcal{O}_{\mathbb{P}^{4}}(1)\right)-E\right)\right)-G\right|$ gives a morphism $\phi: W \rightarrow \mathbb{F}^{1}$ such that the diagram

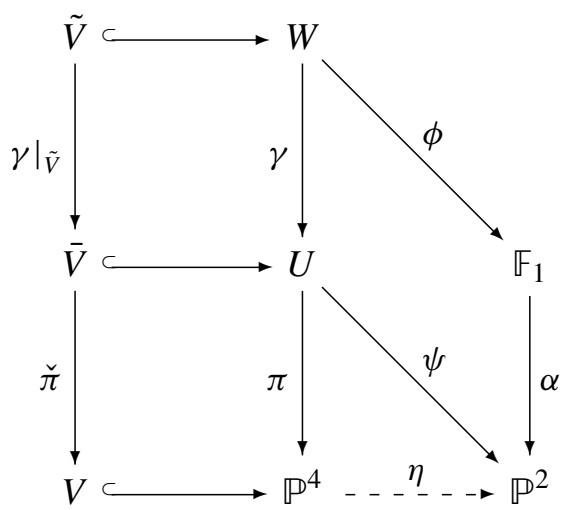

is commutative, where $\alpha: \mathbb{F}^{1} \rightarrow \mathbb{P}^{2}$ is the blow-up of the point $O$. The 3 -fold $\tilde{V}$ is smooth, and the birational morphism $\left.\gamma\right|_{\tilde{V}}$ is a small resolution of the 3 -fold $\bar{V}$.

Lemma 29. The Picard group of the 3 -fold $\tilde{V}$ is $\mathbb{Z} \oplus \mathbb{Z} \oplus \mathbb{Z}$. 
Proof. The divisor $\tilde{V} \subset W$ is rationally-equivalent to a divisor

$$
\begin{aligned}
\gamma^{*}\left(\pi^{*}\left(\mathbb{O}_{\mathbb{P}^{4}}(4)\right)\right. & -2 E)-G \\
& \sim \gamma^{*}\left(\pi^{*}\left(\mathbb{O}_{\mathbb{P}^{4}}(1)\right)-E\right)-G+\gamma^{*}\left(\pi^{*}\left(\mathbb{O}_{\mathbb{P}^{4}}(1)\right)-E\right)+(\pi \circ \gamma)^{*}\left(\mathbb{O}_{\mathbb{P}^{4}}(2)\right)
\end{aligned}
$$

that is ample on the 4-fold $W$. Hence,

$$
H^{2}(\tilde{V}, \mathbb{Z}) \cong H^{2}(W, \mathbb{Z})
$$

by the Lefschetz theorem [Andreotti and Frankel 1959; Bott 1959], which implies the claim of the lemma.

Corollary 30 [Sarkisov 1980]. The restriction $\tilde{\phi}=\left.\phi\right|_{\tilde{V}}: \tilde{V} \rightarrow \mathbb{F}_{1}$ is a standard conic bundle.

Let $\Delta \subset \mathbb{F}_{1}$ be a degeneration divisor of the standard conic bundle $\tilde{\phi}$. Then $\Delta$ is a reduced divisor with at most simple normal crossings [Beauville 1977; Tyurin 1980; Sarkisov 1980, 1982; Shokurov 1983; Corti 2000].

Lemma 31. Let $s_{\infty}$ be an exceptional section of the ruled surface $\mathbb{F}_{1}$, and let $\ell$ be a fiber of the natural projection of the surface $\mathbb{F}_{1}$ to $\mathbb{P}^{1}$. We have

$$
\Delta \sim 5 s_{\infty}+8 \ell \quad \text { and } \quad 2 K_{\mathbb{F}_{1}}+\Delta \sim s_{\infty}+2 \ell .
$$

Proof. Set $\Delta \sim a s_{\infty}+b \ell$ for some integers $a$ and $b$. Consider a general divisor $H$ in the linear system $\left|\tilde{\phi}^{*}(\ell)\right|$ and take the surface $\tilde{\Pi}=\psi^{-1}\left(s_{\infty}\right)$. By Bertini's theorem, $H$ is smooth. The surface $\tilde{\Pi}$ is smooth as well, because

$$
\left.\gamma\right|_{\tilde{\Pi}}: \tilde{\Pi} \rightarrow \bar{\Pi} \cong \Pi
$$

is a blow-up of the four points on $\Pi \cong \mathbb{P}^{2}$ given by $h_{2}=g_{2}=0$.

The birational map $\left.\gamma\right|_{\tilde{\Pi}}$ resolves the base points of the pencil of conics generated by the conics $h_{2}=0$ and $g_{2}=0$, which induces the restriction morphism $\left.\phi\right|_{\tilde{\Pi}}$. The surface $H$ is a cubic surface whose image on the quartic $V$ is a cubic surface residual to the plane $\Pi$. Hence, $K_{H}^{2}=3$ and $K_{\tilde{\Pi}}^{2}=5$, and thus $\Delta \cdot \ell=5$ and $\Delta \cdot s_{\infty}=3$.

The following result is a special case of a conjectured rationality criterion for standard three-dimensional conic bundles [Iskovskikh 1987; 1991; 1996b].

Theorem 32 [Shokurov 1983, Theorem 10.2]. Let $\xi: Y \rightarrow Z$ be a conic bundle such that $Y$ is a smooth 3-fold, $Z$ is either $\mathbb{P}^{2}$ or a ruled surface $\mathbb{E}_{r}$, and $\operatorname{Pic}(Y / Z)=\mathbb{Z}$. If $Y$ is rational and $D$ is a degeneration divisor of $\xi$, then the linear system $\left|2 K_{Z}+D\right|$ is empty.

Therefore, by Theorem 32, the 3-fold $\tilde{V}$ is nonrational, which proves Theorem 6 . 


\section{Acknowledgements}

The author is grateful to A. Corti, M. Grinenko, V. Iskovskikh, S. Kudryavtsev, M. Mella, J. Park, Yu. Prokhorov, A. Pankratiev, A. Pukhlikov, V. Shokurov and L. Wotzlaw for fruitful conversations. The author also thanks the referee for helpful remarks.

\section{References}

[Alekseev 1987] V. A. Alekseev, "On conditions for the rationality of three-folds with a pencil of del Pezzo surfaces of degree 4", Mat. Zametki 41:5 (1987), 724-730, 766. MR 88j:14048 Zbl 0623.14019

[Andreotti and Frankel 1959] A. Andreotti and T. Frankel, "The Lefschetz theorem on hyperplane sections”, Ann. of Math. (2) 69 (1959), 713-717. MR 31 \#1685 Zbl 0115.38405

[Baker 1946] H. F. Baker, A locus with 25920 linear self-transformations, Cambridge Tracts in Mathematics and Mathematical Physics 39, Cambridge, at the University Press, 1946. MR 8,400b Zbl 0063.00170

[Beauville 1977] A. Beauville, "Variétés de Prym et jacobiennes intermédiaires", Ann. Sci. École Norm. Sup. (4) 10:3 (1977), 309-391. MR 57 \#12532 Zbl 0368.14018

[Bese 1983] E. M. Bese, "On the spannedness and very ampleness of certain line bundles on the blow-ups of $\mathbb{P}_{\mathbb{C}}^{2}$ and $\mathbb{F}_{r}$ ", Math. Ann. 262:2 (1983), 225-238. MR 84g:14006 Zbl 0491.14009

[Borcea 1990] C. Borcea, "Nodal quintic threefolds and nodal octic surfaces", Proc. Amer. Math. Soc. 109:3 (1990), 627-635. MR 90k:14038 Zbl 0721.14019

[Bott 1959] R. Bott, "On a theorem of Lefschetz", Michigan Math. J. 6 (1959), 211-216. MR 35 \#6164 Zbl 0113.36502

[Burkhardt 1891] H. Burkhardt, "Untersuchungen aus dem Gebiete der hyperelliptischen Modulfunctionen, II", Math. Ann. 38:2 (1891), 161-224. MR 1510670 JFM 23.0490.01

[Burkhardt 1892] H. Burkhardt, "Untersuchungen aus dem Gebiete der hyperelliptischen Modulfunctionen, III”, Math. Ann. 41:3 (1892), 313-343. MR 1510749 JFM 24.0465.02

[Cheltsov 1997] I. Cheltsov, "On the rationality of non-Gorenstein $\mathbb{Q}$-Fano 3-folds with an integer Fano index", pp. 43-50 in Birational algebraic geometry (Baltimore, MD, 1996), edited by Y. Kawamata and V. V. Shokurov, Contemp. Math. 207, Amer. Math. Soc., Providence, RI, 1997. MR 98i:14040 Zbl 0896.14019

[Cheltsov 2004] I. A. Cheltsov, "Rationality of an Enriques-Fano threefold of genus five", Izv. Ross. Akad. Nauk Ser. Mat. 68:3 (2004), 181-194. MR 2005f:14098 Zbl 1072.14069

[Cheltsov and Park 2004] I. Cheltsov and J. Park, "Sextic double solids", 2004. math.AG/0404452

[Ciliberto and Di Gennaro 2004] C. Ciliberto and V. Di Gennaro, "Factoriality of certain hypersurfaces of $\mathbb{P}^{4}$ with ordinary double points", pp. 1-7 in Algebraic transformation groups and algebraic varieties, edited by V. L. Popov, Encyclopaedia Math. Sci. 132, Springer, Berlin, 2004. MR 2090666 (2005i:14043) Zbl 1068.14010

[Clemens 1975] C. H. Clemens, "Applications of the theory of Prym varieties", pp. 415-421 in Proceedings of the International Congress of Mathematicians (Vancouver, B.C., 1974), vol. 1, Canad. Math. Congress, Montreal, Que., 1975. MR 54 \#10279 Zbl 0405.14017

[Clemens 1983] C. H. Clemens, "Double solids", Advances in Mathematics 47:2 (1983), 107-230. MR 85e:14058 Zbl 0509.14045 
[Clemens and Griffiths 1972] C. H. Clemens and P. A. Griffiths, "The intermediate Jacobian of the cubic threefold”, Ann. of Math. (2) 95 (1972), 281-356. MR 46 \#1796

[Conte and Murre 1977] A. Conte and J. P. Murre, "On quartic threefolds with a double line, I, II", Nederl. Akad. Wetensch. Proc. Ser. A 80 = Indag. Math. 39:3 (1977), 145-160, 161-175. MR 58 \#16686a Zbl 0353.14016

[Corti 1995] A. Corti, "Factoring birational maps of threefolds after Sarkisov", J. Algebraic Geom. 4:2 (1995), 223-254. MR 96c:14013 Zbl 0866.14007

[Corti 2000] A. Corti, "Singularities of linear systems and 3-fold birational geometry", pp. 259-312 in Explicit birational geometry of 3-folds, edited by A. Corti and M. Reid, London Math. Soc. Lecture Note Ser. 281, Cambridge Univ. Press, Cambridge, 2000. MR 2001k:14041 Zbl 0960.14017

[Corti et al. 2000] A. Corti, A. Pukhlikov, and M. Reid, "Fano 3-fold hypersurfaces", pp. 175-258 in Explicit birational geometry of 3-folds, edited by A. Corti and M. Reid, London Math. Soc. Lecture Note Ser. 281, Cambridge Univ. Press, Cambridge, 2000. MR 2001k:14034 Zbl 0960.14020

[Cynk 2001] S. Cynk, "Defect of a nodal hypersurface", Manuscripta Math. 104:3 (2001), 325-331. MR 2002g:14056 Zbl 0983.14017

[Demazure 1980] M. Demazure, "Surfaces de del Pezzo", pp. 21-69 in Séminaire sur les Singularités des Surfaces (Palaiseau, 1976-1977), edited by M. Demazure et al., Lecture Notes in Mathematics 777, Springer, Berlin, 1980. MR 579026 (82d:14021) Zbl 0983.14017

[Dimca 1990] A. Dimca, "Betti numbers of hypersurfaces and defects of linear systems", Duke Math. J. 60:1 (1990), 285-298. MR 91f:14041 Zbl 0729.14017

[Ellia and Franco 2000] P. Ellia and D. Franco, "On smooth surfaces in projective four-space lying on quartic hypersurfaces with isolated singularities", Comm. Algebra 28:12 (2000), 5703-5713. MR 2001m:14056 Zbl 1033.14025

[Endraß 1999] S. Endraß, "On the divisor class group of double solids", Manuscripta Math. 99:3 (1999), 341-358. MR 2000f:14058 Zbl 0970.14006

[Finkelberg 1989] H. Finkelberg, On the geometry of the Burkhardt quartic, Ph.D. thesis, Univ. Leiden, 1989.

[Finkelnberg and Werner 1989] H. Finkelnberg and J. Werner, "Small resolutions of nodal cubic threefolds", Nederl. Akad. Wetensch. Indag. Math. 51:2 (1989), 185-198. MR 90i:14010 Zbl 0703.14026

[Friedman 1986] R. Friedman, "Simultaneous resolution of threefold double points", Math. Ann. 274:4 (1986), 671-689. MR 87k:32035 Zbl 0576.14013

[van der Geer 1987] G. van der Geer, "Note on abelian schemes of level three", Math. Ann. 278:1-4 (1987), 401-408. MR 89a:14053 Zbl 0688.14034

[Grinenko 1998a] M. M. Grinenko, "Birational automorphisms of a three-dimensional double cone", Mat. Sb. 189:7 (1998), 37-52. MR 99i:14018 Zbl 0931.14007

[Grinenko 1998b] M. M. Grinenko, "Birational automorphisms of a three-dimensional dual quadric with the simplest singularity", Mat. Sb. 189:1 (1998), 101-118. MR 99a:14021 Zbl 0934.14007

[Hoffman and Weintraub 2001] J. W. Hoffman and S. H. Weintraub, "The Siegel modular variety of degree two and level three", Trans. Amer. Math. Soc. 353:8 (2001), 3267-3305. MR 2003b:11044 Zbl 0996.11041

[Hulek and Sankaran 2002] K. Hulek and G. K. Sankaran, "The geometry of Siegel modular varieties", pp. 89-156 in Higher dimensional birational geometry (Kyoto, 1997), edited by S. Mori and Y. Miyaoka, Adv. Stud. Pure Math. 35, Math. Soc. Japan, Tokyo, 2002. MR 2003h:11047 Zbl 1074.14021 
[Hunt 1996] B. Hunt, The geometry of some special arithmetic quotients, Lecture Notes in Mathematics 1637, Springer, Berlin, 1996. MR 98c:14033 Zbl 0904.14025

[Iskovskih and Manin 1971] V. A. Iskovskih and J. I. Manin, "Three-dimensional quartics and counterexamples to the Lüroth problem”, Mat. Sb. (N.S.) 86(128) (1971), 140-166. MR 45 \#266 Zbl 0222.14009

[Iskovskikh 1987] V. A. Iskovskikh, “On the rationality problem for conic bundles”, Duke Math. J. 54:2 (1987), 271-294. MR 88i:14038 Zbl 0629.14033

[Iskovskikh 1991] V. A. Iskovskikh, "On the rationality problem for conic bundles”, Mat. Sb. 182:1 (1991), 105-111. MR 92k:14036 Zbl 0776.14013

[Iskovskikh 1996a] V. A. Iskovskikh, "Factorization of birational mappings of rational surfaces from the point of view of Mori theory", Uspekhi Mat. Nauk 51:4(310) (1996), 3-72. MR 97k:14016 Zbl 0914.14005

[Iskovskikh 1996b] V. A. Iskovskikh, “On a rationality criterion for conic bundles”, Mat. Sb. 187:7 (1996), 75-92. MR 97j:14044 Zbl 0922.14026

[Iskovskikh and Prokhorov 1999] V. A. Iskovskikh and Y. G. Prokhorov, "Fano varieties", pp. 1-247 in Algebraic geometry, vol. V, Encyclopaedia Math. Sciences 47, Springer, Berlin, 1999. MR 2000b:14051b Zbl 0912.14013

[Iskovskikh and Pukhlikov 1996] V. A. Iskovskikh and A. V. Pukhlikov, "Birational automorphisms of multidimensional algebraic manifolds", J. Math. Sci. 82:4 (1996), 3528-3613. MR 98e:14009 Zbl 0917.14007

[de Jong et al. 1990] A. J. de Jong, N. I. Shepherd-Barron, and A. Van de Ven, "On the Burkhardt quartic”, Math. Ann. 286:1-3 (1990), 309-328. MR 91f:14038 Zbl 0712.14023

[Kawamata 1988] Y. Kawamata, "Crepant blowing-up of 3-dimensional canonical singularities and its application to degenerations of surfaces", Annals of Mathematics. (2) 127:1 (1988), 93-163. MR 89d:14023 Zbl 0651.14005

[Kollár 1989] J. Kollár, “Flops”, Nagoya Mathematical Journal 113 (1989), 15-36. MR 90e:14011 Zbl 0645.14004

[Kollár 1996] J. Kollár, Rational curves on algebraic varieties, Ergebnisse der Mathematik und ihrer Grenzgebiete (3) 32, Springer, Berlin, 1996. MR 98c:14001 Zbl 0877.14012

[Manin 1967] J. I. Manin, "Rational surfaces over perfect fields, II", Mat. Sb. (N.S.) 72 (114) (1967), 161-192. MR 37 \#1374 Zbl 0182.23701

[Marchisio 2000] M. R. Marchisio, "Unirational quartic hypersurfaces", Boll. Unione Mat. Ital. Sez. B Artic. Ric. Mat. (8) 3:2 (2000), 301-314. MR 2001h:14064 Zbl 0992.14018

[Mella 2004] M. Mella, "Birational geometry of quartic 3-folds, II: The importance of being $\mathbb{Q}$ factorial”, Math. Ann. 330:1 (2004), 107-126. MR 2005h:14030 Zbl 1058.14022

[Petterson 1998] K. F. Petterson, “On nodal determinantal quartic hypersurfaces in $\mathbb{P}^{4}$ ”, 1998, Available at http://folk.uio.no/ranestad/kfpthesis.ps. Thesis.

[Prokhorov 2004] Y. G. Prokhorov, "A remark on Fano threefolds with canonical Gorenstein singularities", pp. 647-657 in The Fano Conference (Turin, 2002), edited by A. Collino et al., Univ. Torino, Turin, 2004. MR 2112596 (2005k:14086) Zbl 1062.14051

[Pukhlikov 1988] A. V. Pukhlikov, "Birational automorphisms of a three-dimensional quartic with a simple singularity", Mat. Sbornik. (N.S.) 135(177):4 (1988), 472-496, 559. MR 89k:14016 Zbl 0668.14007

[Pukhlikov 1998] A. V. Pukhlikov, "Birational automorphisms of three-dimensional algebraic varieties with a pencil of del Pezzo surfaces", Izv. Ross. Akad. Nauk Ser. Mat. 62:1 (1998), 123-164. MR 99f:14016 Zbl 0948.14008 
[Reid 2001] M. Reid, "Graded rings and birational geometry", pp. 1-72 in Sympposium on algebraic geometry (Kinosaki, 2000), edited by K. Ohno, 2001, Available at www.maths.warwick.ac. uk/ miles/3folds/Ki/Ki.pdf.

[Sarkisov 1980] V. G. Sarkisov, "Birational automorphisms of conic bundles", Izv. Akad. Nauk SSSR Ser. Mat. 44:4 (1980), 918-945, 974. MR 82g:14035 Zbl 0453.14017

[Sarkisov 1982] V. G. Sarkisov, "On conic bundle structures", Izv. Akad. Nauk SSSR Ser. Mat. 46:2 (1982), 371-408, 432. MR 84h:14047 Zbl 0593.14034

[Schoen 1985] C. Schoen, "Algebraic cycles on certain desingularized nodal hypersurfaces", Math. Ann. 270:1 (1985), 17-27. MR 86d:14010 Zbl 0533.14002

[Shokurov 1983] V. V. Shokurov, "Prym varieties: theory and applications", Izv. Akad. Nauk SSSR Ser. Mat. 47:4 (1983), 785-855. MR 85e:14040 Zbl 0572.14025

[Todd 1936] J. A. Todd, "On a quartic primal with forty-five nodes, in space of four dimensions", Quart. J. Math. 7 (1936), 168-174. Zbl 0015.04101

[Tyurin 1980] A. N. Tyurin, "The middle Jacobian of three-dimensional varieties", J. Soviet Math. 13 (1980), 707-745. Zbl 0428.14015

[Varchenko 1983] A. N. Varchenko, "Semicontinuity of the spectrum and an upper bound for the number of singular points of the projective hypersurface", Dokl. Akad. Nauk SSSR 270:6 (1983), 1294-1297. MR 85d:32028 Zbl 0537.14003

[Werner 1987] J. Werner, Kleine Aufösungen spezieller dreidimensionaler Varietäten, Bonner Math. Schriften 186, Universität Bonn Mathematisches Institut, 1987. MR 89k:14018 Zbl 0657.14021

Received August 10, 2004. Revised November 21, 2004.

\section{IVAN CHELTSOV}

Steklov Institute of Mathematics

8 GUbKIN STREeT

Moscow 117966

RUSSIA

cheltsov@yahoo.com 